\title{
Keep your partner close
}

It is known that interleukin-15 receptor $\alpha(\underline{\mathrm{IL}-15 \mathrm{R} \alpha)}$ ) presents $\underline{\mathrm{IL}-15}$ in trans to neighbouring cells, and that both IL-15 and IL-15R $\alpha$ support natural killer (NK)-cell homeostasis in resting conditions. However, how this cytokine-receptor pair functions during inflammation is not fully understood. Now, Mortier et al. show that the IL-15-IL-15R $\alpha$ complex is preassembled in dendritic cells (DCs) following Toll-like receptor (TLR) stimulation, and that only membrane-bound complexes, and not secreted complexes, can provide the stimulatory signal necessary for NK-cell activation in vivo.

First, the authors measured the mRNA and protein levels of IL-15 and IL-15R $\alpha$ in DCs following stimulation with TLR ligands. They found increased levels of mRNA expression of both the cytokine and receptor following stimulation, but only detected increased levels of the IL-15R $\alpha$ protein. This led the authors to suggest that the IL-15 protein might be complexed with IL-15R $\alpha$ in DCs following TLR-ligand stimulation, thereby blocking its detection. By developing a new 'complex' ELISA, the authors showed that this was indeed the case. The IL-15-IL-15R $\alpha$ complexes formed in the endoplasmic reticulum or the early Golgi, and IL-15R $\alpha$ was required for the secretion of IL-15 under inflammatory conditions. In addition, both IL-15 and IL-15R $\alpha$ needed to be expressed by the same DC for trans-presentation, refuting the idea that IL- 15 could be secreted by one cell and subsequently bind to IL-15R $\alpha$ on another cell. However, membrane-expressed or secreted IL- $15 R \alpha$ could be detected in the absence of IL-15.

Further analyses showed that the coordinated expression of both IL-15 and IL-15R $\alpha$ specifically by DCs in response to TLR ligands was required for NK-cell activation in vitro or in vivo. However, only membranebound IL-15-IL-15R $\alpha$ complexes, and not the secreted complexes, could activate NK cells and induce interferon- $\gamma$ and granzyme-B production in vivo.

So, the data show that IL-15 and IL-15R $\alpha$ are preformed as complexes in DCs following TLR-ligand stimulation, that IL-15R $\alpha$ acts as a chaperone for IL-15 secretion, as well as a scaffold protein for the transpresentation of IL-15 to NK cells, and that cell-contact between IL-15presenting cells (for example, DCs) and IL-15-responsive cells (for example, NK cells) is required to deliver IL-15-induced signals.

Olive Leavy

ORIGINAL RESEARCH PAPER Mortier, E. Woo, T., Advincula, R., Gozalo, S. \& Ma, A.

IL-15R $\alpha$ chaperones IL-15 to stable dendritic cell membrane complexes that activate NK cells via trans presentation.J. Exp. Med. 205, 1213-1225 (2008)

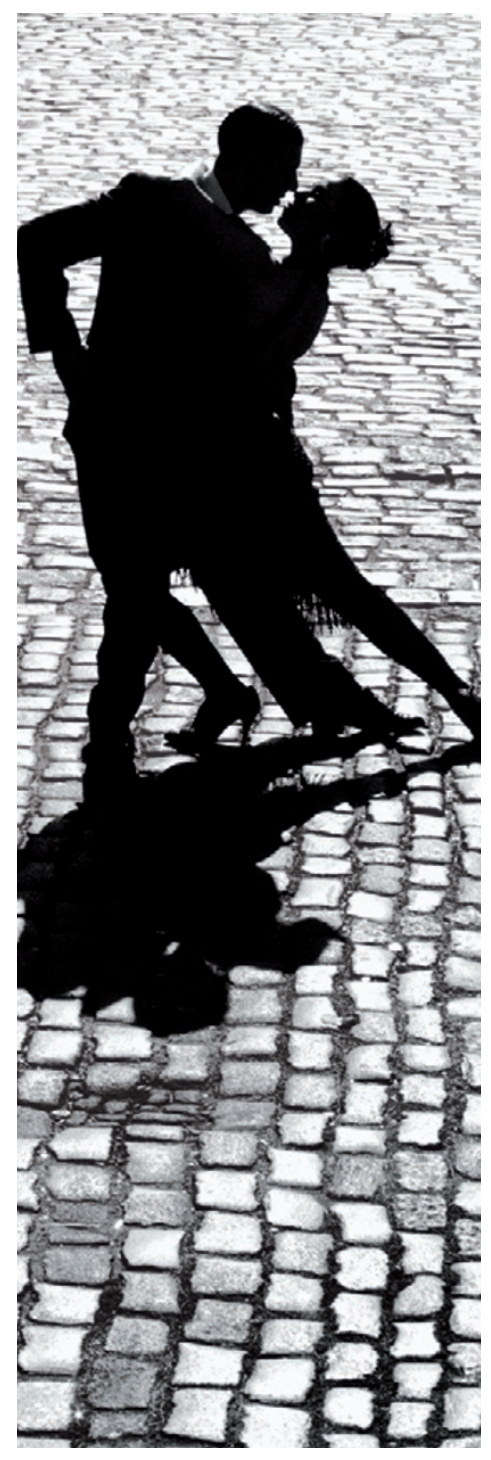

